\title{
The impact of meconium ileus on the clinical course of children with cystic fibrosis
}

\author{
A.K.C. Evans*, D.A. Fitzgerald*,\#, K.O. McKay*,\#
}

The impact of meconium ileus on the clinical course of children with cystic fibrosis. A.K.C. Evans, D.A. Fitzgerald, K.O. McKay. (C) ERS Journals Ltd 2001.

ABSTRACT: The present study was designed to compare the clinical course of children diagnosed with cystic fibrosis $(\mathrm{CF})$ in infancy due to the presence of meconium ileus (MI) with children diagnosed by way of a newborn screening programme (non-MI).

A matched case-control study design was used. Matching was performed on the basis of sex and date of birth. All children born in New South Wales, Australia after 1980 and who had attended the CF clinic at The Children's Hospital at Westmead since diagnosis were included as possible cases or controls. Parameters pertaining to the clinical course were compared in 39 matched pairs.

MI children had a significantly worse pulmonary status. The forced expiratory volume in one second was $16.3 \pm 5.2 \%$ higher $(p<0.001, n=21$ pairs $)$ and the forced vital capacity value $10.5 \pm 4.7 \%$ higher $(p<0.05, n=21$ pairs) in non-MI children. The difference between the pairs (18.6 \pm 4.4 MI and 20.5 \pm 3.4 non-MI) in the Shwachman chest radiograph score was statistically significant $(p<0.05, n=39$ pairs). There were no significant differences in any other assessed parameters, such as height, weight, the presence of liver function abnormalities, the frequency of hospitalization or airway microbial colonization.

Meconium ileus may be an early indication of a more severe phenotype of cystic fibrosis. This was suggested by the significantly lower pulmonary function found in children with a history of meconium ileus compared to age- and sex-matched children who did not have meconium ileus.

Eur Respir J 2001; 18: 784-789.
*Children's Chest Research Centre, Dept of Respiratory Medicine, The Children's Hospital at Westmead (Royal Alexandra Hospital for Children), Westmead, New South Wales, Australia and ${ }^{\#}$ Dept of Paediatrics and Child Health, The University of Sydney, New South Wales, Australia.

Correspondence: K.O. McKay, Dept of Respiratory Medicine, The Children's Hospital at Westmead, Locked Bag 4001, Westmead, New South Wales 2145, Australia.

Fax: 61298453396

\section{Keywords: Cystic fibrosis}

meconium ileus

neonatal screening

nutrition

pulmonary function

\section{Received: June 152000}

Accepted after revision February 20 2001
Diagnosis of cystic fibrosis (CF) is generally made on the basis of either the presence of meconium ileus (MI) in newborns, a positive newborn screening test, suspicion raised by a family history of $\mathrm{CF}$, failure to thrive, or the presence of respiratory and/or gastrointestinal symptoms in childhood [1]. The mode of presentation has been shown to be a significant prognostic factor for survival of patients with $\mathrm{CF}$, as patients presenting with predominantly gastrointestinal symptoms (presumably symptoms such as pancreatitis, steatorrhoea or rectal prolapse) have higher survival rates than those presenting with respiratory symptoms or MI [2].

MI is the presenting feature in $~ 18 \%$ of Australian children with $\mathrm{CF}$, and this incidence is similar to that reported in other countries [3]. There appears to be a tendency for MI to occur in subsequent siblings with $\mathrm{CF}$ when the first child presents with MI [3]. Linkage studies indicate that predisposition to MI may be explained by genetic differences unassociated with mutations of the cystic fibrosis transmembrane regulator (CFTR) gene [4]. Adoption of novel surgical procedures over the last $30 \mathrm{yrs}$, such as the BishopKoop ileostomy, has resulted in a dramatic decrease in the postoperative mortality associated with MI, from $>60 \%$ in the 1960 s to $\sim 0 \%$ in the 1990 s $[5,6]$.

It has been suggested that infants with MI who survive the neonatal period have a long-term survival that is equivalent to children presenting with other symptoms [2]. The earlier diagnosis of $\mathrm{CF}$ in these infants has been suggested as conferring positive prognostic benefits [5]. Moreover, these children have normal lung function at the time of diagnosis [7], and it has been reported that children presenting with MI have less rapid progress of lung disease than children presenting with respiratory symptoms [2]. The absence of long-term effects of MI on nutritional, pulmonary, gastrointestinal and hepatobiliary status has been reported in a number of studies, although some investigators have shown an increase in hepatic abnormalities in patients with a history of MI [8-11].

Therefore, there is evidence that the presence of MI in the newborn period can influence the clinical course of CF. The present authors surmised that this influence may be negative. The absence of control CF groups composed of asymptomatic children also diagnosed in infancy, might have obscured the true long-term effects of MI. This has been reinforced by a recent study, demonstrating a clear association between MI and malnutrition when children diagnosed via newborn screening were used as the control population [12]. The purpose of the present study, which employed a matched case-control study design, was to compare the clinical course of children with $\mathrm{CF}$ 
presenting with MI to the clinical course of children with no history of MI, diagnosed in infancy via newborn screening.

\section{Material and methods}

\section{Study subjects and study design}

This study was approved by the Human Ethics Committee of the Royal Alexandra Hospital for Children, Westmead, New South Wales (NSW), Australia. The medical records of all children attending the $\mathrm{CF}$ clinic at this hospital were scrutinized to identify those that had undergone an annual check in 1999. These children were then further limited to those born in NSW between January 1, 1982-January 1, 1998, who had been followed by this clinic from their birth and who had been diagnosed with $\mathrm{CF}$ on the basis of MI (designated as an MI child). Each MI child (index child) was paired with a child of the same sex and with a birth date in the same month or 1-2 months before or after the index child's birth date. The paired child (non-MI child) had also undergone an annual check in 1999, was also born in NSW during the same period, and had also been followed by the same clinic since diagnosis; however, the paired child was diagnosed as having $\mathrm{CF}$ as a result of newborn screening. Prior to 1994, the newborn screening test consisted of detection of a raised concentration of immunoreactive trypsinogen in a heel-prick blood spot, whereas the test used since 1994 detects the presence of the $\Delta \mathrm{F} 508$ gene mutation. These newborn screening tests were performed on each child born in NSW since August 1981. A confirmatory diagnostic sweat test (Gibson-Cooke) was performed on all children detected via newborn screening, who did not have two copies of the $\Delta \mathrm{F} 508$ gene mutation.

\section{Methods}

Information from the 1999 Annual CF Review, as well as the medical record, was collected for each child. Annual reviews were performed close to the child's birthday and included a physical (respiratory and gastrointestinal) examination as well as an assessment of nutritional, biochemical (liver function tests) and haematological status, sputum cultures and physiotherapy refinement. The physical examination included recording the child's height and weight, as well as the presence of hepatomegaly, splenomegaly, or digital clubbing. Nutritional parameters included serum vitamin A and E levels, vitamin supplementation, pancreatic enzyme replacement therapy and enteral feeding via gastrostomy. The number and duration of hospitalizations and intravenous antibiotic therapy were recorded, as were any episodes of distal intestinal obstruction syndrome and allergic bronchopulmonary aspergillosis. Spirometry was performed on all children aged $>5$ yrs and assessment of lung volumes (by plethysmography) was carried out in children $>8$ yrs. The Shwachman scores [13] for general activity, physical examination, nutrition and chest radiograph, were also recorded.

Height and weight were converted into percentiles, and $\mathrm{z}$-scores (SD scores) and body mass index were calculated. If clubbing was present, the degree of clubbing was recorded using a scale from $1-3$. In this scale, loss of the angle between the nail bed and the cuticle was awarded a score of 1 , convex nails and an increase in the bulk of the terminal pulp was awarded a score of 2, and a score of 3 was awarded in cases where clubbing had progressed to the point where there was an increase in nail curvature and widening of the phalanges. Pancreatic enzyme replacement is expressed as the number of lipase units consumed per $\mathrm{kg}$ of body weight per day. Spirometric values (forced expiratory volume in one second (FEV1), forced vital capacity (FVC) and FEV1/FVC) and lung volumes are expressed as percentage predicted using the normal values published by Polgar and Promadhat [14]. Where known, the genotype was classified as $\Delta \mathrm{F} 508$ homozygous, $\Delta \mathrm{F} 508$ heterozygous or $\Delta \mathrm{F} 508$ null.

\section{Analysis}

As this was a matched case-control study, paired data analysis was performed. Paired t-tests were used for parametric data and the McNemar's test was used for nonparametric variables. A subgroup analysis was performed for the length of stay (in days) and the number of admissions for those patients admitted to hospital, for any reason, in the 12 months prior to their annual review. For this subgroup analysis, comparison was made on a group basis between MI and non-MI patients, using unpaired t-tests. All reported $\mathrm{p}$-values are two-tailed and statistical significance was ascribed to $\mathrm{p}$-values $<0.05$.

\section{Results}

In 1999, 341 patients were seen for an annual review in the CF clinic. Of these, 294 had been born between January 1, 1982-January 1, 1998, and 253 of them had been born in NSW. Of this cohort of 253 children, 39 (22 male) had been diagnosed with $\mathrm{CF}$ due to the presence of MI on the first or second day of life. The matched non-MI patients identified via the newborn screening programme were diagnosed by a positive sweat test at an average age of $60.6 \pm 9.5$ days.

In the MI group, eight children had deceased or living siblings with CF. Two of the children were fraternal twins who both presented with MI. Two children had one younger CF sibling without MI, one child had two younger $\mathrm{CF}$ siblings without $\mathrm{MI}$ and one child had one older and one younger $\mathrm{CF}$ sibling who did not present with MI. One child had a deceased older CF sibling who had not presented with MI and another had a deceased younger CF sibling who had presented with MI. Only in two instances did both or all CF siblings of a family present with MI as newborns.

As expected for the study design, the MI and nonMI patients were well matched for age, as the mean 
Table 1.-Physical characteristics of patients at 1999 annual review

\begin{tabular}{lccc}
\hline & MI group & Non-MI group & Mean difference \\
\hline Age yrs & $9.39 \pm 0.74$ & $9.40 \pm 0.75$ & $-0.02 \pm 0.03$ \\
Height percentile & $43.33 \pm 5.30$ & $28.56 \pm 3.69$ & $14.77 \pm 7.09 *$ \\
Weight percentile & $40.79 \pm 4.67$ & $35.79 \pm 4.28$ & $5.00 \pm 6.58$ \\
Height z-score & $-0.239 \pm 0.197$ & $-0.810 \pm 0.142$ & $0.572 \pm 0.261^{*}$ \\
Weight z-score & $-0.340 \pm 0.159$ & $-0.473 \pm 0.157$ & $0.133 \pm 0.236$ \\
BMI & $16.81 \pm 0.39$ & $17.54 \pm 0.63$ & $-0.731 \pm 0.576$ \\
Shwachman score & $85.13 \pm 1.79$ & $89.49 \pm 1.33$ & $-4.36 \pm 2.37$ \\
\hline
\end{tabular}

Data are presented as mean \pm SEM. BMI: body mass index; MI: meconium ileus. *: $p<0.05$, the mean difference between MI patients and matched non-MI patients is statistically significant using paired t-test, $n=39$ pairs in all cases.

difference in age between the pairs was only 6 days ( $\mathrm{p}>0.05, \mathrm{n}=39$, paired t-test). The diagnostic sweat test results were not significantly different (sweat chloride: $101 \pm 4 \mathrm{mmol} \cdot \mathrm{L}^{-1}$ for MI patients; $100 \pm 3 \mathrm{mmol} \cdot \mathrm{L}^{-1}$ for non-MI patients, $\mathrm{p}>0.05, \mathrm{n}=26$ pairs); however, in 13 pairs of children, one or both of the children had not been sweat tested. Analysis of the children's genotype indicated no statistically significant difference in $\Delta F 508$ homozygosity between children presenting with and without MI. There were 19 pairs of children who were $\Delta \mathrm{F} 508$ homozygous: nine pairs in which only the child with MI was $\Delta \mathrm{F} 508$ homozygous, four pairs in which only the non-MI child was $\Delta \mathrm{F} 508$ homozygous, and six pairs in which neither child was $\Delta$ F508 homozygous ( $\mathrm{p}>0.05, \mathrm{n}=38, \mathrm{McNemar}^{\prime} \mathrm{s}$ test). A similar absence of significant differences was seen in the analysis of $\Delta \mathrm{F} 508$ heterozygosity and cases in which there was no copy of the $\Delta \mathrm{F} 508$ mutation.

The nutritional status of the children was not affected by a history of MI (table 1). The MI children were significantly taller in terms of both height percentile $(14.77 \%$ taller $)$ and height $\mathrm{z}$-scores $(0.572$ greater). The equivalence of the children's nutritional status was also demonstrated by the absence of any significant difference in the nutrition component of the Shwachman score (fig. 1). All the children with MI were pancreatic insufficient and all but two of the

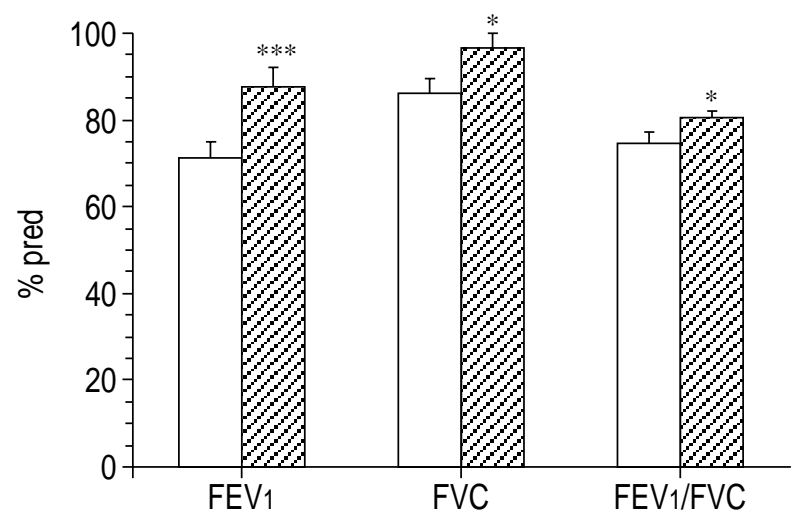

Fig. 1.- Lung function in children with cystic fibrosis with and without a history of meconium ileus (MI). The mean values measured in 21 children with MI in the newborn period (MI, $\square$ ) and their matched pairs with no MI (non-MI, $\mathbb{Z}$ ) are shown and the error bars represent the SEM values. FEV1: forced expiratory volume in one second; FVC: forced vital capacity; \% pred: percentage of predicted values. *: $\mathrm{p}<0.05 ; * * *$ : $\mathrm{p}<0.001$, as assessed by performing paired t-tests. matched controls were pancreatic insufficient, this difference being of no statistical significance ( $p>0.05$, McNemar's). The use of supplemental feeding via gastrostomy was equally distributed in children with and without a history of MI. The children with MI did not require significantly higher levels of pancreatic enzyme replacement therapy than their matched pairs in order to attain and maintain this equivalent nutritional status (mean \pm SEM 4,873 \pm 226 and $4,241 \pm$ 385 lipase units $\cdot \mathrm{kg}$ body weight ${ }^{-1} \cdot \mathrm{day}^{-1}$ respectively, $\mathrm{p}>0.05$, t-test).

A history of MI did not predispose the children to many of the recognized complications of CF. There was no significant difference in the incidence of distal intestinal obstruction syndrome, hepatomegaly, splenomegaly, CF-related diabetes mellitus or allergic bronchopulmonary aspergillosis (all $\mathrm{p}>0.05$, McNemar's, $\mathrm{n}=39$ ) in children with $\mathrm{MI}$ as newborns and their matched pairs. In addition, there were no differences in plasma vitamin $\mathrm{A}$, vitamin $\mathrm{E}$, and nonfasting glucose levels or liver function tests (table 2). The mortality rate during 1999 was no different for children with or without a history of MI. One child died as a result of respiratory failure during 1999; the patient presented with MI as a newborn and was 16.5 yrs old at the time of their death.

Despite having a nutritional status equivalent to their matched pairs, the MI children had a significantly worse pulmonary status. The results of spirometric testing (fig. 2) showed a lower FEV1 $(71.32 \pm 3.86 \%$ and $87.58 \pm 4.77 \%$ pred, MI and nonMI respectively, $\mathrm{n}=21, \mathrm{p}<0.001$, paired t-test), a lower FVC $(86.03 \pm 3.55 \%$ and $96.55 \pm 3.40 \%$ pred, MI and

Table 2.-Plasma biochemistry results at 1999 annual review

\begin{tabular}{lccc}
\hline & MI group & $\begin{array}{c}\text { Non-MI } \\
\text { group }\end{array}$ & $\mathrm{n}$ \\
\hline Vitamin A $\mu \mathrm{mol} \cdot \mathrm{L}^{-1}$ & $1.2 \pm 0.1$ & $1.2 \pm 0.1$ & 39 \\
Vitamin E $\mu \mathrm{mol} \cdot \mathrm{L}^{-1}$ & $11.6 \pm 1.1$ & $14.2 \pm 1.1$ & 39 \\
Nonfasting glucose $\mathrm{mmol} \cdot \mathrm{L}^{-1}$ & $5.6 \pm 0.2$ & $5.3 \pm 0.3$ & 35 \\
Alkaline phosphatase $\mathrm{U} \cdot \mathrm{L}^{-1}$ & $329 \pm 20$ & $306 \pm 19$ & 39 \\
Alanine transaminase $\mathrm{U} \cdot \mathrm{L}^{-1}$ & $37 \pm 4$ & $31 \pm 3$ & 39 \\
Gamma glutamine & $22 \pm 4$ & $21 \pm 3$ & 39 \\
$\quad$ transferase $\mathrm{U} \cdot \mathrm{L}^{-1}$ & & & \\
\hline
\end{tabular}

Data are presented as mean \pm SEM. MI: meconium ileus; $\mathrm{n}$ : number of pairs. There were no statistically significant differences for any measured parameter. 


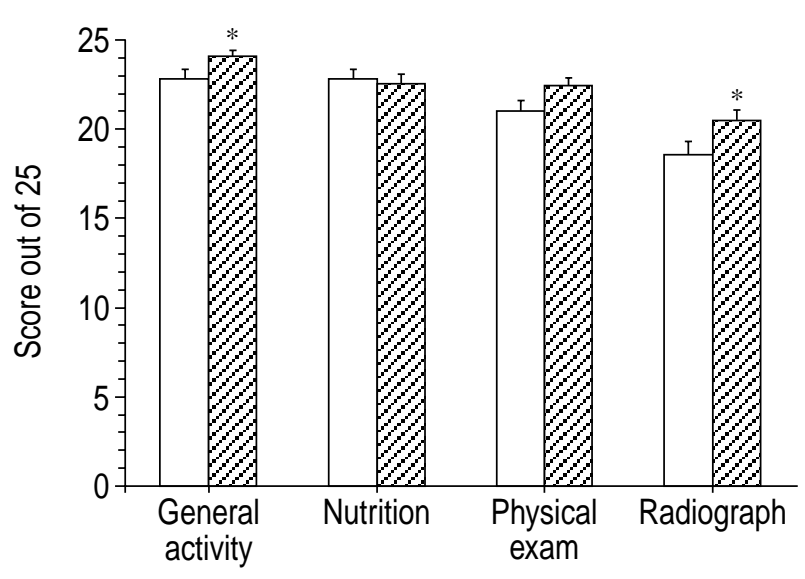

Fig. 2.-Shwachman scores in children with cystic fibrosis with and without a history of meconium ileus (MI). The mean values measured in 39 children with MI in the newborn period (MI, $\square$ ) and their matched pairs with no MI (non-MI, $\mathbb{C}$ ) are shown and the error bars represent the SEM values. *: $\mathrm{p}<0.05$, as assessed by performing paired t-tests.

non-MI respectively, $\mathrm{n}=21, \mathrm{p}<0.05$, paired t-test) and a lower $\mathrm{FEV} 1 / \mathrm{FVC}$ ratio $(74.58 \pm 2.72 \%$ and $80.58 \pm$ $1.49 \%$, MI and non-MI respectively, $\mathrm{n}=21, \mathrm{p}<0.05$, paired t-test). The chest radiograph and the general activity components of the Shwachman score were also significantly lower in these children than that of their matched pairs (fig. 1), but the presence and degree of finger clubbing was not associated with MI in the newborn period. There were 21 pairs of children $>8$ yrs, who underwent lung volume assessment. While there was evidence of a greater residual volume in the MI child in each pair $(181.1 \pm 15.7 \%$ and $161.4 \pm 11.2 \%$ pred $\mathrm{MI}$ and non-MI, respectively), the difference was not statistically significant $(\mathrm{p}>0.05$, t-test, $n=21$ ). The total lung capacity of children with MI was not statistically different from that of non-MI children $(105.9 \pm 6.0 \%$ and $106.8 \pm 2.2 \%$ pred MI and non-MI respectively, $\mathrm{p}>0.05$, t-test, $\mathrm{n}=21$ ).

Airway bacterial colonization patterns were not affected by a history of MI. Sputum cultures from six pairs of children grew normal respiratory flora. There were six instances where only the MI child had normal respiratory flora and another eight where only the non-MI child of the pair was free from colonization with respiratory pathogens. The remaining children were colonized with Staphylococcus aureus or Pseudomonas aeruginosa, with three children having Burkholderia cepacia in their sputum (table 3). In the preceding year, children with a history of MI were more frequently admitted to hospital (MI: $0.54 \pm 0.17$ and non-MI: $0.18 \pm 0.07$ per child in 1999) and required more courses of intravenous antibiotics (MI: $0.41 \pm 0.17$ and non-MI: $0.08 \pm 0.04$ per child in 1999) than non-MI children. However, these differences were not statistically significant $(\mathrm{p}=0.06$ and 0.07 respectively, paired $\mathrm{t}$ tests, $n=39$ ). Subgroup analysis to compare length of stay and the number of admissions in children who had required an admission in the previous year did not reveal statistically significant differences related to a history of MI.
Table 3. - Sputum culture results for 1999 for children with and without a history of meconium ileus (MI) as newborns

\begin{tabular}{lccc}
\hline & $\begin{array}{c}\text { Staphylococcus } \\
\text { aureus }\end{array}$ & $\begin{array}{c}\text { Pseudomonas } \\
\text { aeruginosa }\end{array}$ & $\begin{array}{c}\text { Burkholderia } \\
\text { cepacia }\end{array}$ \\
\hline $\begin{array}{l}\text { Both children } \\
\text { in pair }\end{array}$ & 2 & 8 & 1 \\
$\begin{array}{l}\text { MI child only } \\
\text { Non-MI child } \\
\text { only }\end{array}$ & 7 & 9 & 0 \\
$\begin{array}{c}\text { Neither child } \\
\text { in pair }\end{array}$ & 21 & 4 & 1 \\
\hline
\end{tabular}

\section{Discussion}

This study confirms that MI in the newborn period does not affect the long-term nutritional status of children with CF. MI may, however, be an early indication of a more severe form of CF. This is suggested by the detection of significantly lower pulmonary status in children with a history of MI than in age- and sex-matched children who did not have MI as newborns.

The observation of a decreased pulmonary status (as measured by lung function testing and chest radiography) in the children presenting with MI in this study, supports the suggestion that MI may be an indicator of a more severe variant of $\mathrm{CF}$ [15]. Although the residual volumes were not significantly higher in the children with a history of MI, this may reflect the small number of pairs of children who were old enough to perform these manoeuvres. Nevertheless, the spirometric measures and chest radiograph scores were all lower in children who had MI. This finding is contrary to that reported in the study of FUCHS and LANGER [9], which showed no significant difference in respiratory status in patients who had presented with MI, and age- and sex-matched patients.

There are a number of reasons for the lack of consensus between the results of the present study and that of FuCHS and LANGER [9]. In the present study, the children with MI were matched with children who had also been diagnosed in infancy by way of a statewide newborn screening programme. The matched children in the study of FUCHS and LANGER [9], however, were presumably diagnosed on the basis of symptomatology, as the mean age at diagnosis was 7.7 yrs. As it has been shown that the early detection of $\mathrm{CF}$ reduces morbidity $[15,16]$ and promotes superior nutritional status later in life [17], it is likely that the matched control children in the present study had benefited from early detection of their disease.

Abnormalities in pulmonary function have been reported in very young infants with $\mathrm{CF}$ diagnosed on the basis of clinical symptoms [7], but lung function appears to have been normal in asymptomatic infants [18]. Thus, it is conceivable that these early abnormalities partly explain why previous studies have shown that MI in the newborn period had no effect upon lung function later in life. Abnormalities in pulmonary function in the non-MI population used as controls, may have masked the true impact of MI. 
This assertion is supported by the work of WATERS et al. [19], who demonstrated superior pulmonary function at both 5 and $10 \mathrm{yrs}$ of age in children diagnosed via a newborn screening programme. By using matched controls also diagnosed in infancy, but by way of newborn screening before symptoms could develop, the present study has been able to eliminate these masking effects. The matched control children were diagnosed at approximately the same age as the children with MI, were all born and grew up in the same state, and have been treated within the same clinic using the same treatment strategies. By adopting this study design, some of the confounding variables have been eliminated, thus allowing the effects of MI on the clinical course of children with CF to be more accurately delineated.

It is possible that the impaired pulmonary status associated with a history of MI could be explained by the $\mathrm{CF}$ genotype of the patients. Relationships between the CF genotype and the presence of MI have been found. The G551D and R117H mutations are associated with a decreased incidence of MI, while there is a positive association between MI and the $\Delta$ F508 and G542X mutations [20-22]. Moreover, very young children, homozygous for the $\Delta \mathrm{F} 508$ mutation and diagnosed via newborn screening, have been shown to have early evidence of airway obstruction when compared with children of other genotypes [23]. Despite this, studies of older children have shown that while poor pulmonary function may be related to pancreatic insufficiency [24], it is not influenced by the CF genotype $[20,21]$. Therefore, the CF genotype is unlikely to explain the present results. No significant difference in pancreatic insufficiency or genotype was found between the matched pairs. There were, however, nine pairs in which the MI child alone was $\Delta \mathrm{F} 508$ homozygous. Therefore, the genotype of the second mutation in the non-MI children may, in part, explain the differences in lung function. The limited population available for study and the disparity in second genotypes precludes firm conclusions in this regard.

Studies of sibling pairs with CF and identical CFTR genotypes have indicated the presence of modifier genes for MI on chromosome 19 [25]. Hence, it is possible that modifier genes could explain the presence of more severe pulmonary disease in children with a history of MI. Future studies may, therefore, affirm or eliminate a role for modifier genes in determining pulmonary status of patients with $\mathrm{CF}$, with and without a history of MI.

TEPPER et al. [7] showed that at the time of diagnosis, infants presenting with MI have a lung function that is indistinguishable from healthy control infants. Therefore, the relative decrement in pulmonary status associated with MI is not likely to have been a result of any in utero pulmonary abnormalities associated with the development of bowel obstruction in these children. This contention is supported by evidence of normal pulmonary anatomy and histology in children with a history of MI [26, 27]. The point at which the relative decrement in pulmonary status first becomes obvious is yet to be revealed by serial lung function studies in children with MI and matched controls. Because the present study included lung function assessments on children $\geqslant 5$ yrs of age, the authors conclude that the decrement in lung function must develop sometime between infancy and 5 yrs of age.

The results of the present study suggest that meconium ileus is an indication of a more severe phenotype of cystic fibrosis, which may be conferred by the presence or absence of particular modifier genes. While nutrition is unaffected by meconium ileus later in life, adverse pulmonary status is related to the presence of this complication. Greater surveillance of, and early attention to, the pulmonary status of these patients may assist in preserving their lung function.

\footnotetext{
Acknowledgements. The annual checks performed for this study were a collaborative effort by all members of the Cystic Fibrosis team at The Children's Hospital, Westmead, New South Wales. The authors would like to thank M. Winner, M. Gray and the other staff of the Medical Records Dept for their valuable assistance. Financial assistance was provided by way of a scholarship from the Amulree Elective Bursary Committee for A. Evans to travel to Australia from University College, London, UK.
}

\section{References}

1. Rosenstein BJ, Cutting GR. The diagnosis of cystic fibrosis: A consensus statement. J Pediatr 1998; 132: 589-595.

2. Hudson I, Phelan PD. Are sex, age at diagnosis, or mode of presentation prognostic factors for cystic fibrosis? Pediatr Pulmonol 1987; 3: 288-297.

3. Allan JL, Robbie M, Phelan PD, Danks DM. Familial occurrence of meconium ileus. Eur J Pediatr 1981; 135: 291-292.

4. Mornet E, Simon-Buoy B, Serre JL, et al. Genetic differences between cystic fibrosis with and without meconium ileus. Lancet 1988; 1: 376-378.

5. McPartlin JF, Dickson JAS, Swain VAJ. Meconium ileus. Immediate and long-term survival. Arch Dis Child 1972; 47: 207-210.

6. Del Pin CA, Czyrko C, Ziegler MM, Scanlin TF, Bishop HC. Management and survival of meconium ileus. A 30-year review. Ann Surg 1992; 215: 179-185.

7. Tepper RS, Hiatt P, Eigen H, Scott P, Grosfeld J, Cohen M. Infants with cystic fibrosis: pulmonary function at diagnosis. Pediatr Pulmonol 1988; 5: 15-18.

8. Kerem E, Corey M, Kerem B, Durie P, Tsui L-C, Levison $\mathrm{H}$. Clinical and genetic comparisons of patients with cystic fibrosis, with or without meconium ileus. J Pediatr 1989; 114: 767-773.

9. Fuchs JR, Langer JC. Long-term outcome after neonatal meconium obstruction. Pediatrics 1998; 101: e7.

10. Colombo C, Apostolo MG, Ferrari M, et al. Analysis of risk factors for the development of liver disease associated with cystic fibrosis. J Pediatr 1994; 124: 393-399.

11. Maurage C, Lenaerts C, Weber A, et al. Meconium ileus and its equivalent as a risk factor for the development of cirrhosis: An autopsy study in cystic fibrosis. J Pediatr Gastroenterol Nutr 1989; 9: 17-20. 
12. Lai H-C, Kosorok MR, Laxova A, Davis LA, FitzSimmon SC, Farrell PM. Nutritional status of patients with cystic fibrosis with meconium ileus: A comparison with patients without meconium ileus and diagnosed early through neonatal screening. Pediatrics 2000; 105: 53-61.

13. Shwachman H, Kulczycki L. Long-term study of one hundred and five patients with cystic fibrosis. Am J Dis Child 1958; 96: 6-29.

14. Polgar G, Promadhat V. Pulmonary Function Testing in Children: Techniques and Standards. Philadelphia, WB Saunders Co., 1971.

15. Wilcken B, Chalmers G. Reduced morbidity in patients with cystic fibrosis detected by neonatal screening. Lancet 1985; ii: 1319-1321.

16. Dankert-Roelse JE, te Meerman GJ, Martijn A, ten Kate LP, Knol K. Survival and clinical outcome in patients with cystic fibrosis, with or without neonatal screening. J Pediatr 1989; 114: 362-367.

17. Farrell PM, Kosorok MR, Laxova A, et al. Nutritional benefits of neonatal screening for cystic fibrosis. N Engl J Med 1997; 337: 963-969.

18. Godfrey S, Mearns M, Howlett G. Serial lung function studies in cystic fibrosis in the first 5 years of life. Arch Dis Child 1978; 53: 83-85.

19. Waters DL, Wilcken B, Irwig L, et al. Clinical outcomes of newborn screening for cystic fibrosis. Arch Dis Child Fetal Neonatal Ed 1999; 20: F1-F7.

20. Hamosh A, King TM, Rosenstein BJ, et al. Cystic fibrosis patients bearing both the common missense mutation Gly $\rightarrow$ Asp at codon 551 and the $\Delta \mathrm{F} 508$ mutation are clinically indistinguishable from $\Delta \mathrm{F} 508$ homozygotes except for decreased risk of meconium ileus. Am J Hum Genet 1992; 51: 245-250.

21. The Cystic Fibrosis Genotype-Phenotype Consortium. Correlation between genotype and phenotype in patients with cystic fibrosis. N Engl J Med 1993; 329: 1308-1313.

22. Feingold J, Guilloud-Bataille M. Genetic comparisons of patients with cystic fibrosis with or without meconium ileus. Ann Genet 1999; 42: 147-150.

23. Mohon RT, Wagener JS, Abman SH, Seltzer WK, Accurso FJ. Relationship of genotype to early pulmonary function in infants with cystic fibrosis identified through newborn screening. J Pediatr 1993; 122: 550555.

24. Kerem E, Corey M, Kerem B-S, et al. The relation between genotype and phenotype in cystic fibrosis - analysis of the most common mutation $(\Delta \mathrm{F} 508)$. $N$ Engl J Med 1990; 323: 1517-1522.

25. Zielenski J, Corey M, Rozmahel R, et al. Detection of a cystic fibrosis modifier locus for meconium ileus on human chromosome 19q13. Nat Genet 1999; 22: 128-129.

26. Zuelzer WW, Newton WA Jr. The pathogenesis of fibrocystic disease of the pancreas. A study of 36 cases with special reference to the pulmonary lesions. Pediatrics 1949; 4: 53-69.

27. Sturgess J, Imrie J. Quantitative evaluation of the development of tracheal submucosal glands in infants with cystic fibrosis and control infants. Am J Pathol 1982; 106: 303-311. 